\title{
Automatic Generation of Computer Models through the Integration of Production Systems Design Software Tools
}

\author{
Pavel Vik ${ }^{1}$, Dias Luís, Pereira Guilherme and José Oliveira \\ University of Minho, Centro ALGORITMI, Campus de Gualtar, Braga, 4710 - 057, Portugal
}

Received 15 May 2010, Accepted 20 September 2010

\begin{abstract}
The design of production and logistic systems is a process of managing both technical and organizational variants in order to identify the best solution for a given system. This is a very well-known industrial engineering issue, where the objectives for designing such a system have been changing over the last decades. Former approaches were concerned about material handling costs only but more recent works include re-layout and product mix costs, together with a great concern on processes - high service levels, optimal scheduling policies, setup times and costs, etc. Nowadays, the rapid technological progress and the associated competitive problems lead to a great need of fast and successful solutions to deal with continuous change (re-design) of the currently used industrial systems. Flexibility, modularity, efficiency and robustness are generally highly desired system properties. For general design of industrial systems, three basic types of software tools are used: Computer Aided Design, Simulation and Information Systems. These tools help on improving the utilization of system resources like equipment, manpower, materials, space, energy, information, etc. Nevertheless these three types of software tools have been used with low levels of integration. This absence of an adequate data connection and integration of outputs cause time delays in the design process, duplication of work and could also be a source of errors. In this work, Production Systems Design software tools integration possibilities are discussed and a unified system architecture solution, implemented on AutoCAD (layout design), Witness (Simulation) and MS-Access (Information Systems) is presented. The aim is to focus on the need of data coherence between different software tools, exploring ways of dealing with data diversity and assuring valid and efficient solutions. MS-Access supports the specification of the system and data exchange between Witness and AutoCAD. Based on the database specification, our application automatically generates simulation programs and also different spatial patterns of project layouts. These tasks are implemented in Visual Basic code. Iteratively the results from the simulations are used to improve AutoCAD layouts and AutoCAD layouts are used in new simulations. The use of our application, in the examples showed in this paper, proved to get quick, valid and efficient solutions.
\end{abstract}

Key words: production system design, computer simulation, CAD system, database system, automatic generated simulation models, automatic generated layouts.

\section{Introduction}

This paper describes an internal logistic system in the automotive industry - namely a manufacturer of plastic parts for cars. Its production and logistic system is strictly based on customer requirements and it is very hard to establish optimal solution and a static optimal system configuration. For this purposes, it is common to use computer simulation which can be used in many ways.

Constructing a simulation model is time consuming. Adapting a previous simulation model could also be time consuming. However simulation proved to be an important tool to deal with production and logistic systems design. In this project we propose a way of automatically generating simulation models. We also propose a way of automatically generating layout designs. Furthermore we propose to integrate both approaches and iteratively get better solutions for our production and logistic system. Both tools would interact with a suggested database that would be able to correctly describe the whole system under study. Advantages and disadvantages are then discussed. The problem under analysis in this paper has been previously approached by the conventional way models manually constructed (Jareš 9); The approach propo- sed in this paper - models created automatically, is part of our current research work (Vik 19, 20, 21).

Assembly lines in the automotive industry produce hundreds of cars per day. It is critical to supply the correct component to the correct line at the appropriate time. Nowadays mass customization leads to the possibility of choosing a car according to specific customer requirements. This impels companies to provide a very large set of product variants. It is then possible to reach over 50000 real variants for a particular type of car - virtually more than 8 billion different possible combinations.

To tackle the problem of large quantities of product variants, and due essentially to capital and storage limitations, production strategies have emerged - JIT (Just in Time) or even JIS (Just in Sequence). These strategies imply that components are just supplied to production line when they are needed (Hutchins 8), so reducing storages and buffers. These production approaches make it possible to anticipate to suppliers an exact production schedule, exact production sequences, etc.

Nevertheless models developed in this work do include the possibility of changing production sequences (as a conse-

Corresponding author: vikpavel@seznam.cz 
quence of changing assembling sequences) in the internal logistic system, combining production and storage areas.

Logistics of production systems became one of the most important issues in the automotive industry. Companies in this area don't differ too much in technology and equipment used. Essentially quality of products and services, especially logistic services would make the difference. Critical aspects for a successful company would then include:

- flexibility to customers' requirements

- rapid adaptation to customers' requirements

- permanent flexibility to actual market needs

- permanent need of quality increasing

- short time reactions to any type of changes

The time pressure in the design of production systems and frequent changes mentioned above leads to our suggested approach of currently used software tools integration in the area of production systems design. These tools would refer to project data analysis, design and consequent validation, namely:

\section{- Databases}

- CAD systems

- Discrete simulation

These tools are usually used with low levels of integration, leading to redundant work and absence of data coherence. Our approach supports the integration of a CAD system with a simulation tool through a common database together with some developed functions for data exchange.

This integration approach has the following aims:

- Designing a unique database for a set of different projects, with all detailed production system behaviour specified

- Constructing automatic functions (for data analysis, for generating simulation models, for generating layout alternatives)

- Avoiding redundant project work

- Reducing sources of human errors (in design, in modelling, in coding, etc.). This reduction is due to integrated system coherence and use of automatic model generators.

- $\quad$ Easy feedback processing

- Producing specific documents for an easy balance of different alternatives

- $\quad$ Standardizing design phase

Traditional use of simulation can then be divided into the following steps (phases):

1. Definition of project tasks

- Definition of exact project targets

- Decision of using computer simulation as the solving tool

- Setting of system's boundaries and level of detail

- $\quad$ Team building and its responsibility

2. Processing and obtaining data
- Technical data (facilities data, product data, information about material flows, production areas, breakdowns etc.)

- Organizational data (production scheduling)

- Business data (costs, orders)

3. Creation of the simulation model

- Conceptual model (schematic)

- Interlacing model

- Computer model

4. Simulation run and experiments

- Setting of parameters (e.g. length of simulation run)

- Model validation and verification

5. Interpretation of results and implementation

- Data evaluation

- Interpretation of results and presentation (graphs, tables, animation)

This method is based on "Systematic layout planning" developed by Muther 15, Zelenka [23], Francis et al. 6

Our project shows some possibilities of saving time through the automatic generation of solutions, especially in these phases:

- Creation of the simulation model

- Layout design (CAD drawing)

- Presentation of results (statistical graphs, display of material flows in layout (Havlík et al. 7)

\section{Project Description}

This project describes a part of the internal logistic system, in the production of plastic car parts (bumpers). The simple schema of production is shown in Fig. 1.

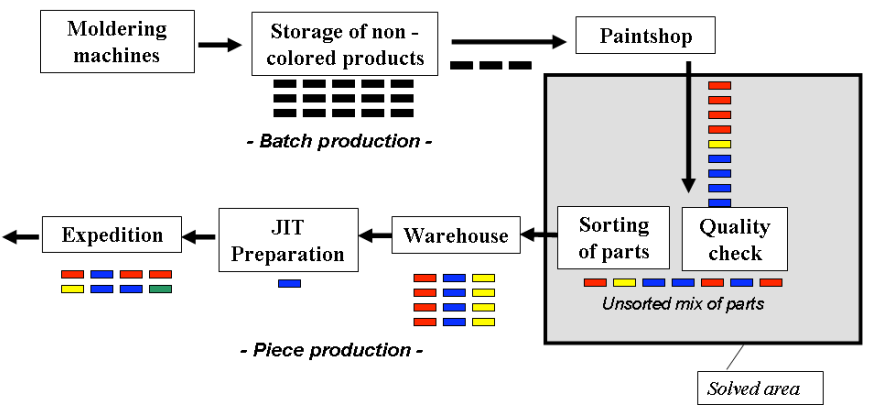

Fig. 1. Schema of production processes

Injection molding machines produce non-coloured bumpers and these are consequently painted in paint shop. Bumpers are divided according to the frequency of their production into large-lot (around $80 \%$ of production) and small-lot production (20\% - mainly some unusual colours). This is the reason why there are two paint shop lines for each type of production. After painting, bumpers are transported by conveyors into quality checking places. Five different classifications apply - perfect (around 65\% of the production), locally repairable by brushing (15\%), able to local repainting (15\%), need of complete repainting (5\%) and scrap product $(5 \%)$. Perfect and repairable products are hitched back to conveyor. At the end of the conveyor the products are put into transport boxes according to shape and colour. Transport boxes (crates) are different based on shape of bumpers and they have also different capacities. Crates are stored in the warehouse. From 
the warehouse, the parts are delivered do assembly workshop according to JIT system demands.

Production consists of over two hundred variants of bumpers depending on shapes (front or back, car type) and colours. In this area, it is fundamental to use a good logistic system, mainly because of the following reasons:

- $\quad$ Large range of product types (two hundred specific variants);

- $\quad$ High daily production (thousands of products);

- Strict JIT customer demands (time between ordering and supplying is around four hours), each final product after assembly has unique properties (type and colour of main bumper parts, combined with assembled components as holder of identification mark, lights, parking sensors etc.);

- Batch Production in the paint shop is stocked in an intermediate super market to supply the assembly task that behaves following a JIT production philosophy depending on customer orders;

Specific area studied

This paper focuses on a specific area of the factory - modelled and studied through simulation. It is the area between the paint shop and the warehouse. Painted parts leave paint shop in sorted batch sequences, respective quality is checked, and the good ones are transported on a conveyor next to the warehouse. Then they are hung down and stored into transport crates in the warehouse. Parts arrive randomly and it is then necessary to have a temporary storage for crates not full.

Tasks

The main objective for this work is to find out the best way for a systematic adaptation to new customer requirements, introducing the adequate changes in production and suppliers This paper then discusses possible changes on the current logistic system (transportation system, storage system and material flow).

The following parameters were identified, to which special attention should be driven:

- Number of operators to hang down bumpers from the conveyor

- Number of transport boxes and size of storage space

- Number of fork-lifts

- Size of temporary storage area (capacity) where transport boxes (partially filled) are stored

- Brush workplaces

Solving

The main tool for performing with these tasks is Discrete Computer Simulation, useful to:

- Deal with the stochastic system behaviour (product quality, processing times, batch sequences)

- Gain easy testing and evaluating alternatives

- Reach the ability to deal with large and complex systems

The following chapters would then describe two different approaches for solving the same type of task and would compare them, showing advantages and disadvantages - in fact, the main focus of this paper.

\section{Manually created models - conventional way}

For solving the given tasks, the following software tools were used:

- MS Excel 2003 as a data storage tool

- Witness 2008 as a simulation tool (Dias et al. 5, Markt et al. 11)

- AutoCAD 2004 as a layout design tool

It was developed according to the following phases:

1) Definition of project tasks

2) Processing and obtaining input data

3) Creation of simulation model

4) Simulation run and experiments

5) Interpretation of results and implementation

\subsection{Definition of project tasks and input data (phase 1 and 2)}

Project tasks specification is identical for both approaches as well as input data:

- Technological processes and operations specification

- Part routes in the studied area

- Production scenarios (output sequences of bumpers from paint shop, defining colour and shape of each bumper, transport box capacity, etc.)

- Stochastic behaviour for bumpers according to quality level and checking performing times. These data were obtained from company databases (monitoring each workplace and its outputs)

- Size of temporary storage (buffer) for transport boxes

- $\quad$ Statistic data for fork-lifts - measuring of transport times

\subsection{Creation of simulation model (phase 3)}

A conceptual model was then created, with description of inputs, outputs and logic control (Fig. 2).

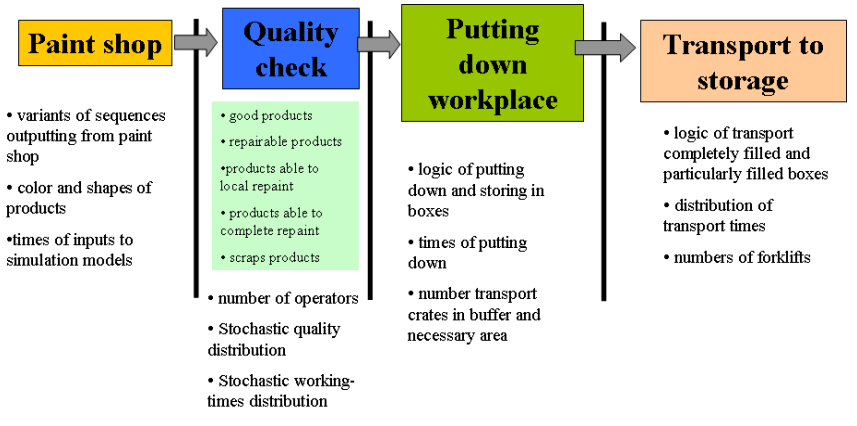

Fig. 2. Conceptual model

According to this conceptual model a simulation model was developed. Fig. 3 shows a part of this model created in Witness 2008 simulation tool. 


\subsection{Simulation run and experiments (phase 4)}

Several experiments were performed and feedback from system's reactions was monitored. Different scenarios of paint shop production scheduling were tested according to different tact times of paint shop and different outputting sequences. The experiments performed also tested different values for some parameters e.g. incrementing/decrementing time out for moving partially filled boxes, changing number of operators, forklifts etc.

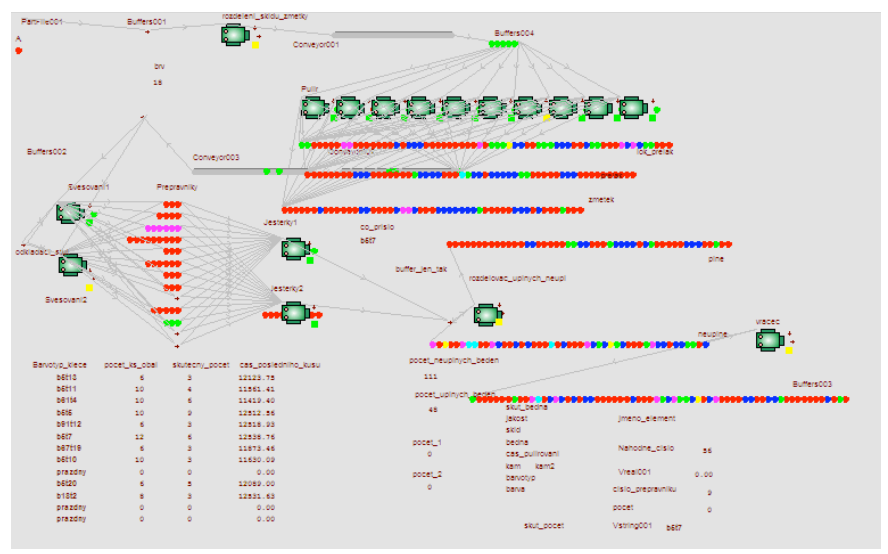

Fig. 3. Screenshot of simulation model and example of simulation results

\subsection{Interpretation of results and implementation}

Simulation model was created to evaluate all important parameters of the real system. Simulation results have shown that current production system would be adequate by simply changing some parameters and that changes in process logic control, capacities or space would not be necessary. These results (e.g. utilization of operators, forklifts or storage area) for each sequence were compared and it was found that the structure of any sequence does not influence the functionality of the system. Fig. 3 shows some results.

\section{Automatic models generation - new ap- proach}

The essential part of this approach consists of the integration of software tools, commonly used in systems design (Moorthy [12], Mecklenburg [11], Aleisa et al. [1]) Database system; CAD system; and Computer Simulation. Fig. 4 shows the purpose of each tool and also the interconnection possibilities.

Similar approach is used in "holistic" systems called "digital factories" (Bureš et al. [3], Chen et al [4], Neil [16])The kernel of our approach is the database (MS Access 2003) which is the storage for all project data and also includes functions for its analysis and functions for generation of computer simulation programs in Witness Simulation tool 2008 .

Simulation model and experiments make it possible numerically evaluate and compare different scenarios which give important results such as utilization of system resources, material flows, buffers occupation etc. The results are automatically updated in specific tables of the database. That data is then used in the automatic generation of production system layout in CAD tool (AutoCAD 2004). In such a layout it is possible to identify the position of facilities (using alternative icons symbols or accurate facility drawing); material flows (with different arrows accordingly to actual flows from simulation results); entities; and specific place for storages and buffers, etc. (Benjaafar [2], Kulturel [10], Sly [17], Moorthy [13]).

Following chapters describe details of each tool and integration functions.

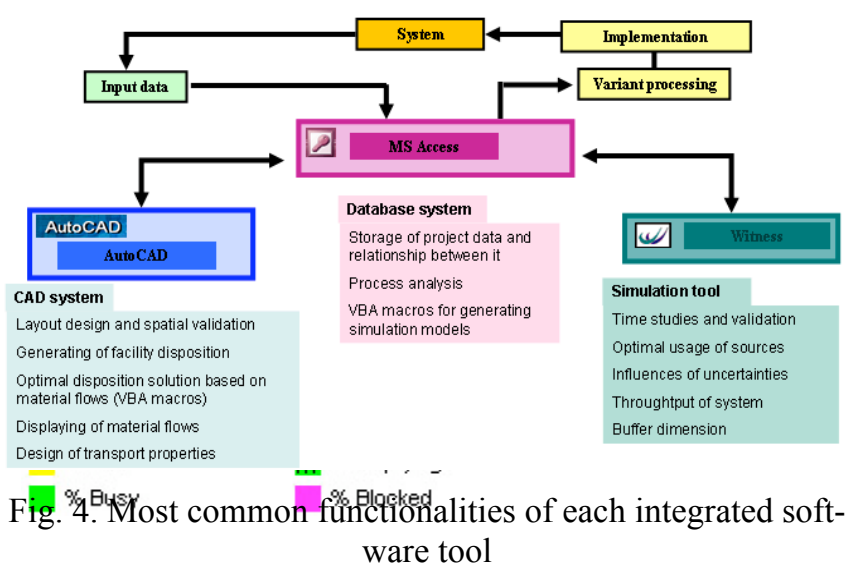

\subsection{Database system}

The system database (MS Access) serves as the source and repository for project data (with input and output data from simulation and CAD), and it builds a basic interconnection element. The database includes VBA macros for automatic generation of simulation elements (machines, buffers, etc.) and their properties with minimum user intervention.

So, this database connects all necessary project data by appropriate relationship between them.

The required data for the simulation model is (Taylor [18]):

- Definition of operations (input and output parts/entities, batches sizes, assembly information, process times, etc.);

- Production sequences of parts/entities (i.e. their routing through production facilities);

- Type of crates and transported quantity;

- Productive and non-productive facilities (machines and handling equipment) and their properties (setup times, transport speeds, etc.);

- Scheduling of production;

- Workers and their properties.

Fig. 5 displays a set of selected tables (nearly half of the database), representations and relationships from the relational database. For example, inputs for operations, material flow between stations and table of priorities for each operation and process are described in the database.

In the top right corner of this screenshot there are three tables "ListOfInventory", related to "Library_Blocks_process" by the table "Tbl_CAD_Inventory". This association assigns different AutoCAD drawings to each element in the inventory, which are representations for system resources or production 
facilities. Connected to "ListOfInventory" we can find the table "Tbl_working_possible" that relates the resources with "ListOfOperation", associating feasible operations for each resource/facility. Table "ListOfOperation" includes a list of operations and some properties of the operations like production times, setup times, possible production facility locations, etc. Each operation has input (table "Inputs") and output (table "Outputs") definition, describing incoming and outgoing parts, quantities (batch sizes), type of container (crate) used, etc. There are also tables to define parts/entities ("ListOfParts") and transport crates ("ListOfCrates"), also with relationship with CAD data tables ("Library_blocks_part", "Library_blocks_crate") that includes references and properties of CAD drawing blocks. There is similar structure for definition of space, and also some other specific tables describing material flows, production scheduling, human resources, tables including results from simulation (statistics) and from CAD layout (coordinates for objects).

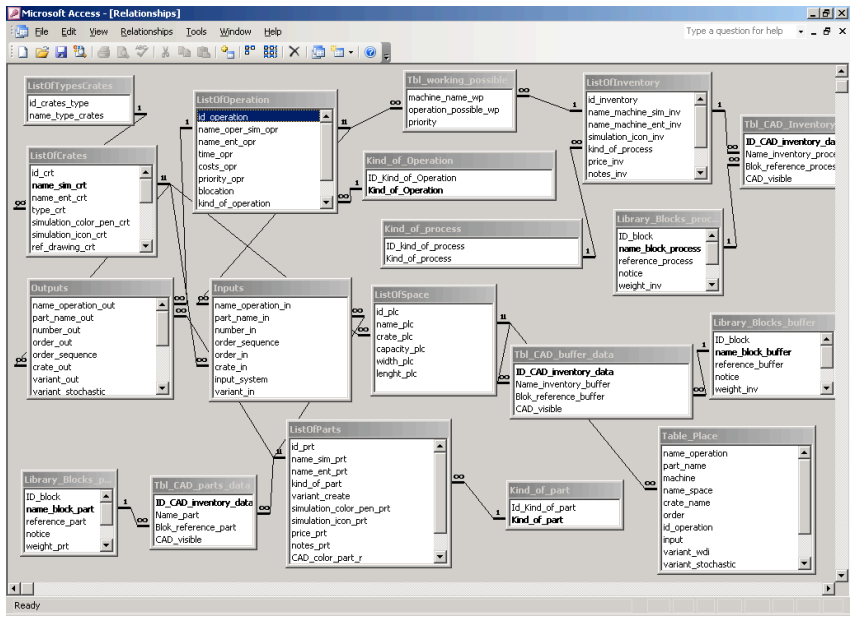

Fig. 5. Snapshot from MS Access relational database

The database, moreover, contains VBA macros for automatic loading of CAD objects into AutoCAD - also with minimum operator intervention - and elements of fundamental analysis (mainly PQ analysis helps to identify the most important product family and also optimal layout type - flow line, flow shop, job shop, etc.).

Material flow analysis from-to table and simple heuristic optimization methods based on material flow values identify optimal location of system resources. Cluster analysis helps finding similar sequences of technological processes (and designing production cells or production lines).

\subsection{Simulation System}

The generated simulation model consists of the previously mentioned elements and other elements such as lists (operations, facilities, parts, etc.), modules for project database establishment and modules involving SQL queries for data loading. These modules automatically feed simulation elements with appropriate data, configuring and updating them. The system can also configure the simulation runs according to database information (performing model's initialization in the beginning of each simulation run). As a consequence, users only need to change data in the database directly or through user-friendly forms in order to manage the simulation model.

After the generation and configuration of the model, subsequent simulation runs and experimentations are performed, and the obtained data are recorded back to the database. Output data are mainly results of stochastic behaviour in material flows, average and maximum occupation of buffers (this information is used in design of the CAD layout), resources utilization (machines, operators and handling equipment), bottlenecks, system throughputs, etc.

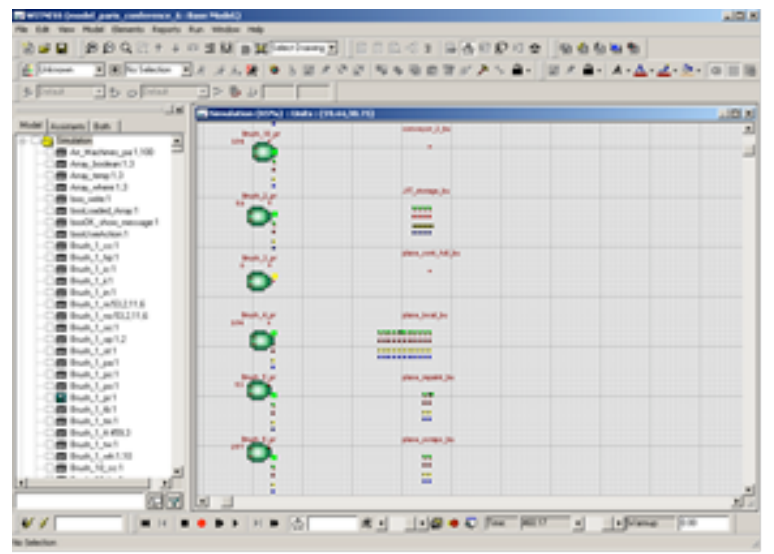

Fig. 6. Illustration of Witness simulation model

Fig. 6 contains a screenshot of a generated simulation model - actual occupation of buffers (queues), utilization of facilities (pie graphs) and a list of additional information (list of facilities, parts, and operations) (Mujber [14] and Vilarinho[22])

\subsection{CAD System}

The CAD system (Autodesk AutoCAD v. 2004) is mainly used for identifying best locations for all facilities and displaying them in the selected layout. For this purpose, VBA macros enable the identification of the optimal positions, currently according to a simple constructive heuristic method - a triangular algorithm based on ranking of material flow data.

The detailed layout is influenced by several factors and limitations (e.g. current production facilities or building constraints).

Fig. 6 shows an automatically generated layout (left side of figure) of a system with material flows of specific entities (ideal and real). Each machine/operation is represented by a small rectangle followed by the output buffer (blue rectangles). Flows are represented by line segments with different widths accordingly to the actual entities movements evaluated numerically in the simulated model (in witness). In fact, actual material flow is a major criterion for selection of the best solution. In the

Fig. 6 (right side), there is a layout limited by constraints (current production facilities, architectonics limitation as walls, pillars and others). 


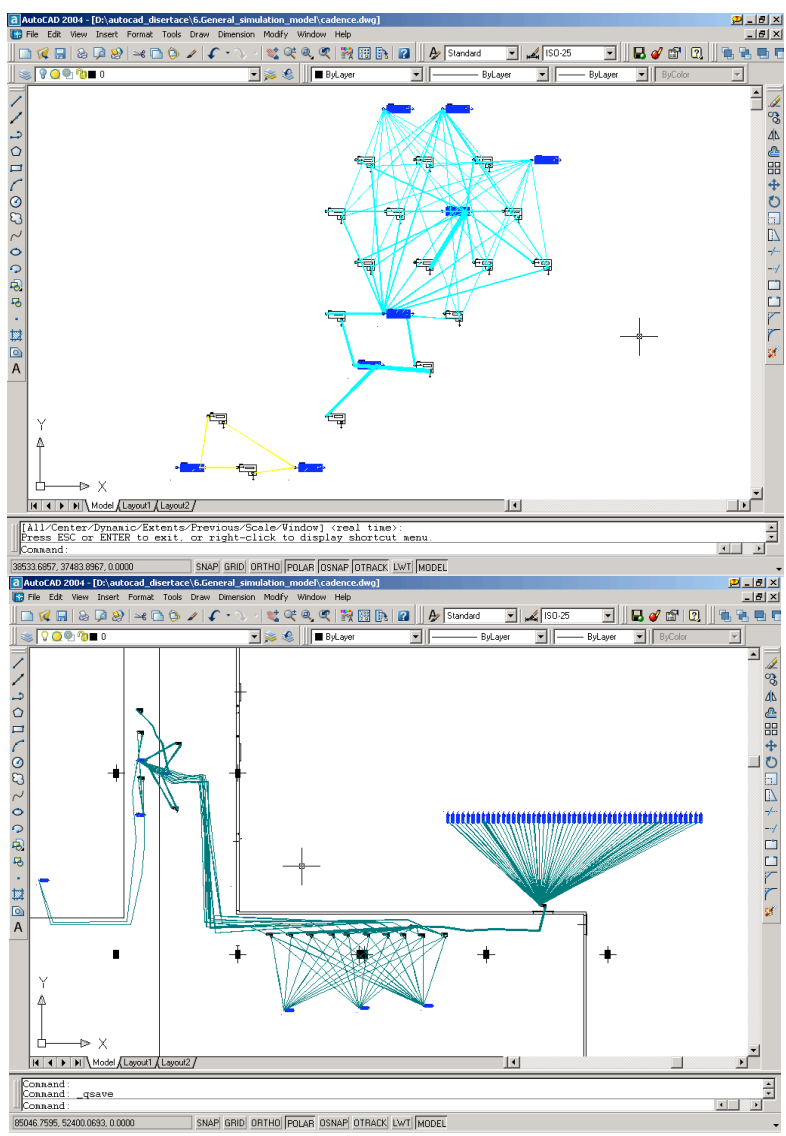

Fig. 6. Snapshots of layout from AutoCAD for a model with material flows (ideal and real)

Information related to material flows can have different units (e.g. weight transported in a time period or frequency of transport movement). Flows can be displayed according to various parameters - related to specific parts, to pallet transportation or to any transport facility.

\section{Comparing both approaches}

We described two approaches for solving the same set of tasks - a model constructed manually by the user and a model automatically generated by a solution tool integration. In this Chapter we compare them in different ways:

- Results

- $\quad$ Speed and time requirements

- Changing of settings and application of feedbacks

- Explanation of results, clearness, graphics

- Documentation

\subsection{Results}

These values are related to the same facilities in the manually created model and the automatically generated one. As far as facilities utilizations statistics are concerned (

Fig. 7), results are very similar, with differences around 5\% (which could be acceptable due to stochastic system behaviour).

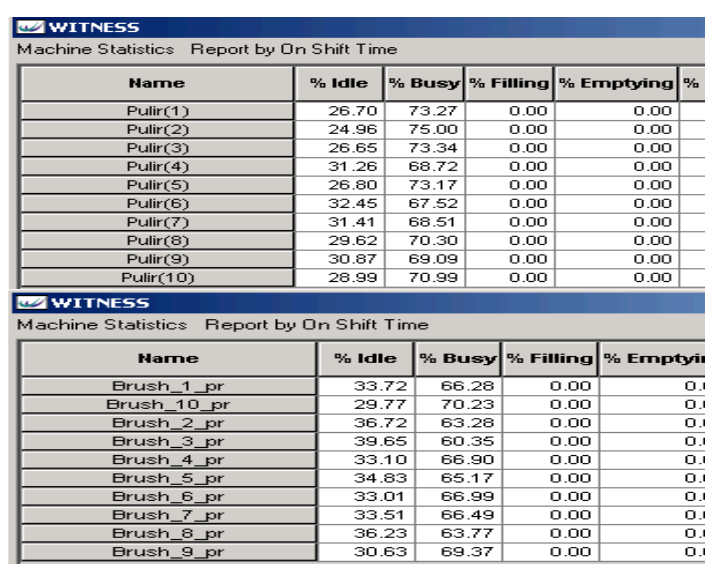

Fig. 7. Comparing of results

\subsection{Time requirements for creating simulation model}

Manual creation of the model took around 2 days of a simulation practitioner. Automatic generation took only a couple of minutes.

Another function of this integrated solution that contributes to saving time is the generation of facility layout in CAD once drawing blocks (e.g. facilities) are automatically inserted into drawing and also material flows between facilities are represented.

Fig. 6 shows some simple blocks representing real facilities. Material flows are distinguished in AutoCAD by layers and colours and displayed according to several factors - product type, used crate (box, pallets etc.) or a sum of flows between facilities.

\subsection{Changing of settings and application of feed- backs}

As shown on example of production times, the Fig. 8 shows two ways of changing a specific cycle time. In the manually created model, user must find an element and change cycle time property in Witness (Fig. 8, left side)

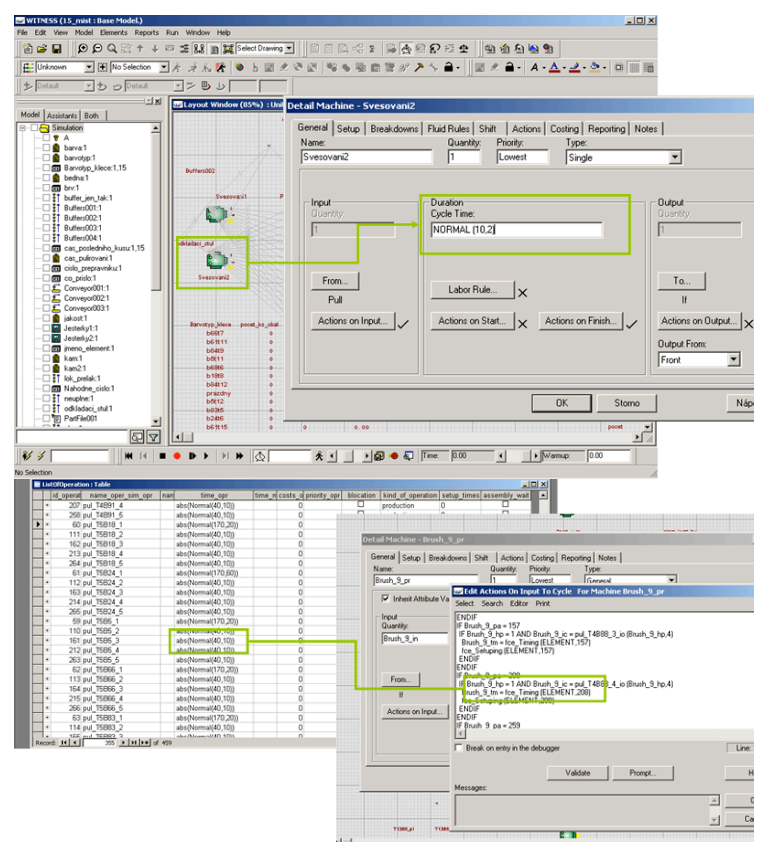

Fig. 8. Changing of settings 
In the integrated approach with simulation model automatic generation, information is changed in project database; all model data are in one common database and it is easy to changing it (Fig. 8, right side). If the data are changed, simulation model is automatically updated in the initialization phase of next simulation run. Or if it is necessary (as by adding a new object), a new model would be generated.

\subsection{Explanation of results, clearness, graphic}

Generated simulation model is not built for animation view; it is mainly for "background" simulation and it is not as clear as in the case of manually created models. On the other hand, generated models contain some other functions for making results more clear. These functions are used automatically, without user intervention. For example:

- Maximum occupation for each product in the buffers (Fig. 9 - left side)

- Occupation - time graphs for estimating storage size (Fig. 9 - right side)

- Table with process results - waiting times, setup times and also a list of processed operation (their name, number and time) for each facility (Fig.10)
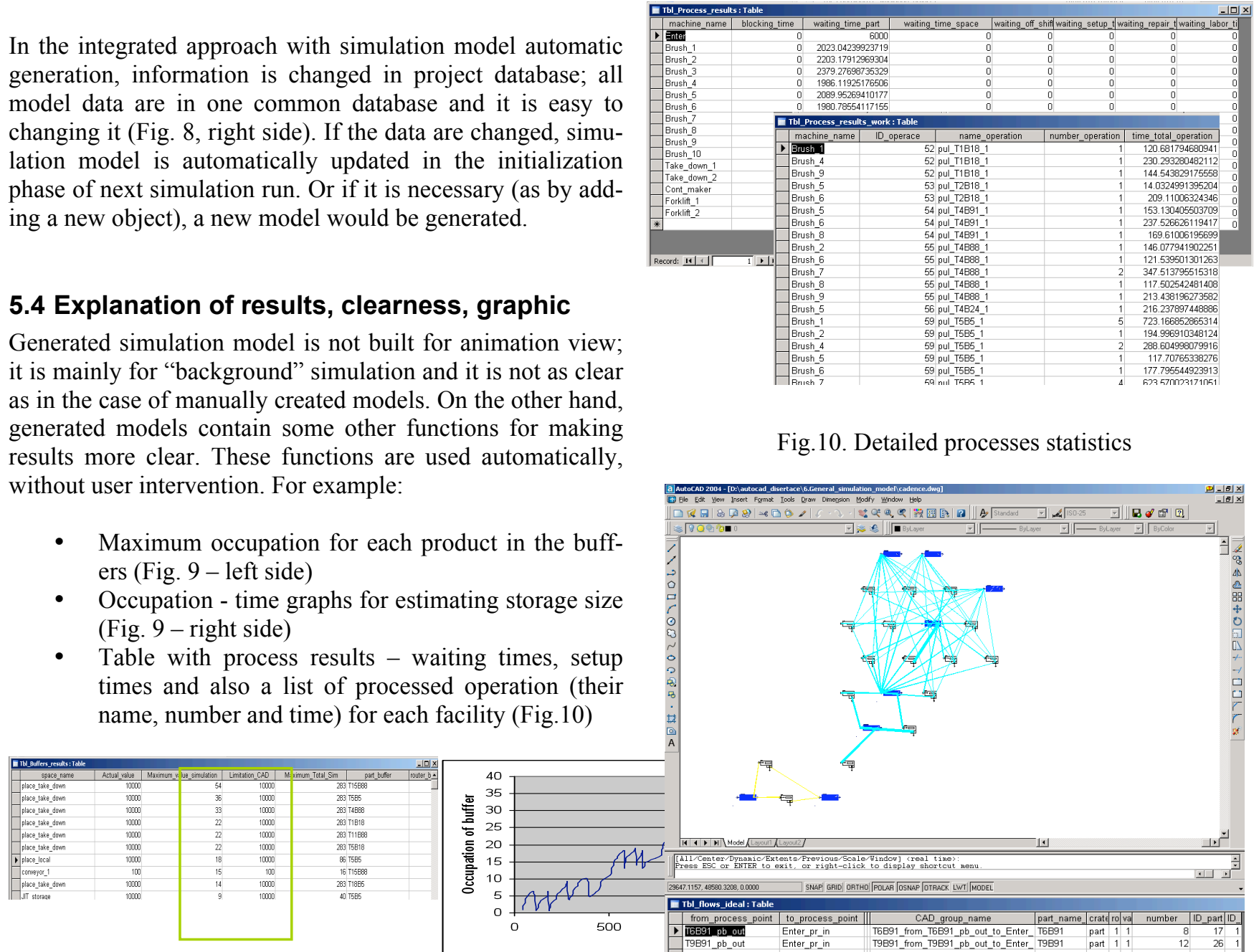

Fig.10. Detailed processes statistics

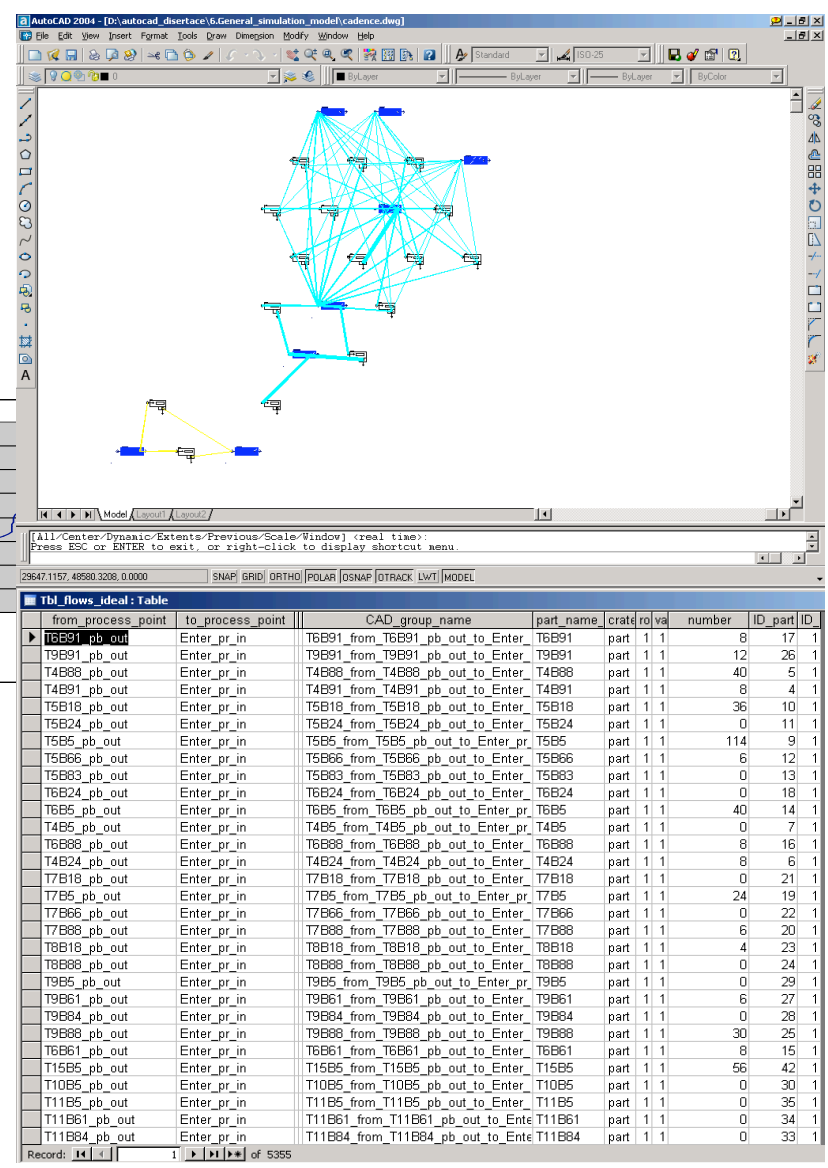

Fig. 11. Displaying of material flows two production facilities. Different colors of lines identify different part types (or type of crate used) and it is possible to filter them by switching on/off their AutoCAD layers. Material flow information helps for better facilities positioning - it helps to realize which facilities must be closer to each other.

\section{Conclusion}

At the end, both approaches would best be adequate for certain project phases where the following fundamental advantages would apply:

The phases of designing production systems can be divided into the following four phases, according to "Systematic layout design" developed by Richard Muther 15:

1. Location - determination of space and another important resources

2. Overall layout - arrangement of areas and transport aisles 
3. Detail layout - determination of specific machinery and equipment

4. Installation - detailed specification of the system

For the first two phases (overall system design) of a production system design project, the automatic generation of models would be appropriate. Mainly because of the following reasons:

- To avoid fundamental errors in early project phases

- The fast creation of models and immediate results to be used in the comparison of different alternatives, enable choosing good solutions rapidly

- Included feedbacks support configuration changes very quickly

- The connection to a common database for simulation tool and CAD system allows to work on all system resources together and also to work on the overall system optimization

In detail layout project phase it seems to be better to use models manually created. Mainly for these reasons:

- Detailed logic control (like in complex material flows)

- Specific 3D space limits (instead of the 2D limitations of the automated approach)

- Refined 3D animation (for better visualization of the designed system). Could be useful for some specific issue like check 3D collision of moving entities.

- Concerning of another specific factors like human ergonomics, vibration, noise, pollution etc.

\section{References}

1. Aleisa E., Lin L., For effective facilities planning: layout optimization then simulation, or vice versa? In: Proceedings of the Winter Simulation Conference, 1381-1385, (2005)

2. Benjaafar, S., Heragu, S. S., Irani S., Next generation Factory layouts: Research challenges and recent progress, Interface (2002)

3. Bureš M., Ulrych Z.,Leeder E. Digitální fabrikasoftwarové produkty pro oblast digitální fabriky, Mopp - Modelování a optimalizace podnikový procesů, Plzeň 8.-9. 2. ISBN 978-80-7043-535-9 (2007)

4. Chen D., Kjellberg T., Euler A. Software Tools for the Digital Factory - An Evaluation and Discussion, Proceedings of the 6th CIRP-Sponsored International Conference on Digital Enterprise Technology, Springer Berlin, ISSN1615-3871 (2009)

5. Dias, L.,Pereira, G. and Rodrigues, G., A Shortlist of the Most Popular Discrete Simulation Tools, Simulation News Europe, 17(1), 33-36, ISSN 0929-2268 (April 2007)

6. Francis R.L., McGinnis L.F., White J.A., Facility Layout and Location: An Analytical Approach, 2nd edition, Prentice-Hall, Englewood Clifs, NJ, USA. (1992)
7. Havlík, R.; Manlig, F., Vom 2D Layout bis zur virtuellen Realität, Priemyselne inženierstvo. Sborník prŕspěvků mezinárodní konference, Dolný Smokovec 07.-08.10.04., 2004, Košice: TU v Košiciach,

8. Hutchins D., Just in Time, Gower Publishing Company, ISBN 978-0566077982 (1999)

9. Jareš, D. Počítačová simulace transportního systému v podniku $X$, Liberec. Bachelor work (2007)

10. Kulturel S., Approaches to uncertainties in facility layout problems: Perspectives at the beginning of the 21th Century, Springer Science and Business Media, Springer Netherlands, ISSN 0956-5515 (2007)

11. Markt L.,Mayer M., Witness simulation software a flexible suite of simulation tools, Proceedings of the 1997 Winter Simulation Conference, 711-717 (1997)

12. Mecklenburg K., Seamless integration of layout and simulation, Proceedings of 2001 Winter Simulation Conference, 1487- 1491.

13. Moorthy S., Integrating the CAD model with $d y$ namic simulation: simulation data exchange, Proceedings of the 1999 Winter Simulation Conference, 276-281 (1999)

14. Mujber T.S., Szecsi T., Hashmi M., A new hybrid dynamic modelling approach for process planning, Journal of Materials Processing Technology 167, (2005)

15. Muther R. Systematické projektováni (SLP), SNTL, Praha (1973)

16. Neil S., The Digital factory, Managing Automation (USA), 22(10), (2007)

17. Sly David P., A systematic approach to factory layout and design with Factoryplan, Factoryopt and Factoryflow, Proceedings of the Winter Simulation Conference, 584-587, (1996)

18. Taylor G. Introduction to logistics engineering, New York, Taylor \& Francis Group (2008)

19. Vik P., Integration of CAD systems and computer simulation and its usage in designing of manufacturing systems, 3. mezinárodní konference Výrobní systémy dnes a zítra, 27-28.11.2008

20. Vik P., Dias L., Pereira G., Software Tools Integration for the Design of Manufacturing Systems, Industrial Simulation Conference 2009, June 1-3, 2009, Loughborough,UK, ISBN 978-90-77381-4-89

21. Vik P., Dias L., Pereira G., Integration possibilities of software tools used in design of manufacturing systems, ACC Journal. XV, (2009) ISSN 1803-9782

22. Vilarinho P., Guimaraes R., A Facility Layout Design Support System, Investigacao Operacional 23, 145-161, (2003)

23. Zelenka A., Král M., Projektováni výrobnich systémů, Praha: ČVUT, 1995,ISBN80-01-013302-2 